\title{
REFERENCES
}

1. M. L. Cartwright, Some decomposition theorems for certain invariant continua and their minimal sets, Fund. Math. vol. 48 (1960) pp. 229-250.

2. W. H. Gottschalk and G. A. Hedlund, Topological dynamics, Amer. Math. Soc. Colloquium Publications, Vol. 36, 1955.

3. W. H. Gottschalk, Minimal sets: An introduction to topological dynamics, Bull. Amer. Math. Soc. vol. 64 (1958) pp. 336-351.

UNIVERSITY OF DELAWARE AND University of Cambridge

\section{THE KRONECKER PRODUCT OF GRAPHS}

\section{PAUL M. WEICHSEL}

Introduction. This note considers a graph product derived from the Kronecker product of matrices. Some indication of the geometrical nature of this product is given and a theorem stating necessary and sufficient conditions for a graph product to be connected is proved. The matrix analogue of the above result is also stated.

I. A convenient representation for a finite undirected graph [1] $G$ is an adjacency matrix. If the vertex set of $G$ is $\left\{p_{i}\right\}, i=1, \cdots, n$, then an adjacency matrix of $G$ is an $n \times n$ matrix $\left(a_{i j}\right)$ with $a_{i j}$ equal to the number of lines (paths of length one) joining $p_{i}$ to $p_{j}$. A given graph is then represented by an equivalence class of matrices $\bar{A}=\left\{P_{i} A P_{i}^{-1} \mid\right.$ for all permutation matrices $P_{i}$ of order equal to the order of $A\}$. Each element of $\bar{A}$ corresponds to a different ordering of the vertices of $G$. It is clear that for each such class of adjacency matrices there corresponds a unique class of isomorphic graphs.

From this point on "graph" will mean a finite undirected graph with no loops. Such a graph has an adjacency matrix $\left(a_{i j}\right)$ whose entries are non-negative integers such that $a_{i j}=a_{j i}$ and $a_{i i}=0$. We also use the following notation: $o(G)$ is the number of vertices of $G$ and is called the order of $G, p_{1} \rightarrow p_{k}$ is a chain in $G$ from vertex $p_{1}$ to vertex $p_{k}$, and $n\left(p_{1} \rightarrow p_{k}\right)$ is the number of lines (not necessarily distinct) in $p_{1} \rightarrow p_{k}$.

If $A$ and $B$ are two adjacency matrices and $A \circ B$ is some matrix product which is also an adjacency matrix then this matrix operation

Presented to the Society, September 1, 1961; received by the editors November 20, 1960. 
induces a composition of the graphs represented by $A$ and $B$. In order that a given matrix product induce an appropriate graph product the following conditions must be satisfied.

(1) $A \circ B$ should be an adjacency matrix of some graph.

(2) For any permutation matrices $P_{1}$ and $P_{2}$ of appropriate orders there exists a permutation matrix $P$ such that:

$$
P(A \circ B) P^{-1}=\left(P_{1} A P_{1}^{-1}\right) \circ\left(P_{2} B P_{2}^{-1}\right) \text {. }
$$

That is, the matrices $A \circ B$ and $\left(P_{1} A P_{1}^{-1}\right) \circ\left(P_{2} B P_{2}^{-1}\right)$ are equivalent in the sense defined above.

Two other properties are desirable.

(3) $\overline{A \circ B}=\overline{B \circ A}$ and

(4) $\overline{(A \circ B) \circ C}=\overline{A \circ(B \circ C) \text {. }}$

One matrix operation which satisfies all of the above requirements is the Kronecker product. Hence we may define the Kronecker product of two graphs as follows:

Definition 1. Let $A$ and $B$ be adjacency matrices of the graphs $G$ and $H$ respectively. The Kronecker product, $G \otimes H$, is that graph with adjacency matrix $A \otimes B$, the Kronecker product of $A$ and $B$.

Notice that condition (2) above guarantees that regardless of the choice of $A$ and $B$ the graph associated with $A \otimes B$ will be the same up to isomorphism.

The Kronecker product of two graphs can also be represented in a somewhat more direct manner.

Definition $1^{\prime}$. Let the vertex sets of the graphs $G$ and $H$ be $\left\{p_{i}\right\}, i=1, \cdots, n$, and $\left\{q_{j}\right\}, j=1, \cdots, m$, then $G \otimes H$ is a graph with vertex set $\left\{p_{i} q_{j} \mid p_{i} q_{j}=q_{j} p_{i}\right.$ for $\left.i=1, \cdots, n, j=1, \cdots, m\right\}$ such that the adjacency number of the pair $p_{i} q_{j}, p_{k} q_{l}$ is the product of the adjacency numbers of $p_{i}, p_{k}$ in $G$ and $q_{j}, q_{l}$ in $H$.

It is easy to show that Definition 1 and Definition $1^{\prime}$ are equivalent.

As an illustration of the geometrical properties of the Kronecker product consider the product of two stars. A star is a graph with one vertex singly adjacent to all other vertices and no other adjacencies. Clearly such a graph has an adjacency matrix $\left(a_{i j}\right)$ with $a_{1 j}=a_{i 1}=1$ for $i, j \neq 1$ and $a_{i j}=0$ otherwise. If $G$ and $H$ are stars of orders $a$ and $b$ respectively then $G \otimes H$ is a graph with adjacency matrix

$$
\left(\begin{array}{ll}
C & 0 \\
0 & D
\end{array}\right),
$$

where $C$ is an adjacency matrix of a star of order $(a-1)(b-1)+1$ and $D$ is an adjacency matrix of a graph whose vertex set can be 
partitioned into two sets containing $a-1$ and $b-1$ vertices respectively such that each vertex is adjacent to every vertex not in its set and to no others. This can be shown either by applying an appropriate sequence of permutations to the rows and columns of the Kronecker product of adjacency matrices or by listing the vertices of $G \otimes H$ and deriving the adjacency relations by the criterion of Definition $1^{\prime}$. The graph defined by $D$ can also be described as a "star" with $a-1$ "endpoints" and $b-1$ "centers." For example, let $G$ and $H$ be stars of orders 6 and 4 respectively. Then
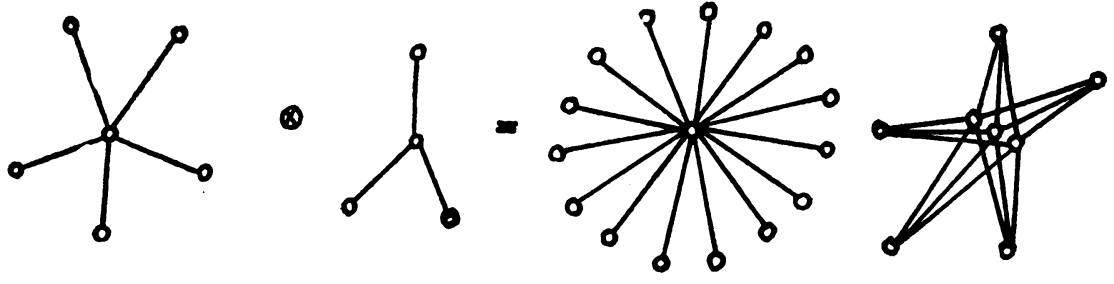

Figure 1

Notice that the product of these two graphs is a disconnected graph. We now examine the question as to when the Kronecker product of two graphs is connected.

Lemma 1. Let $G$ and $H$ be graphs with vertex sets $\left\{p_{i}\right\}$ and $\left\{q_{j}\right\}$ respectively. If $p_{1} q_{1}$ and $p_{2} q_{2}$ are two vertices of $G \otimes H$ and if there exist $p_{1} \rightarrow p_{2}$ in $G$ and $q_{1} \rightarrow q_{2}$ in $H$ such that $n\left(p_{1} \rightarrow p_{2}\right)+n\left(q_{1} \rightarrow q_{2}\right)$ is even then there exists $p_{1} q_{1} \rightarrow p_{2} q_{2}$ in $G \otimes H$.

PRoof. Suppose $n\left(p_{1} \rightarrow p_{2}\right) \leqq n\left(q_{1} \rightarrow q_{2}\right)$. Then there exists $p_{1} q_{1} \rightarrow p_{2} q_{k}$ in $G \otimes H$ for some $q_{k}$ in $H$ and the path $q_{k} \rightarrow q_{2}$ has an even number of lines. If $p_{t}$ is a vertex adjacent to $p_{2}$ in $G$ and $\left(q_{k}, q_{k+1}, \cdots, q_{2}\right)$ $=q_{k} \rightarrow q_{2}$ then the path $\left(p_{2} q_{k}, p_{t} q_{k+1}, p_{2} q_{k+2}, \cdots, p_{2} q_{2}\right)$ connects $p_{2} q_{k}$ to $p_{2} q_{2}$. Hence the composite of $p_{1} q_{1} \rightarrow p_{2} q_{k}$ and $p_{2} q_{k} \rightarrow p_{2} q_{2}$ connects $p_{1} q_{1}$ to $p_{2} q_{2}$ in $G \otimes H$.

A cycle of a graph is called odd if it contains an odd number of vertices.

TheOREM 1. Let $G$ and $H$ be connected graphs. $G \otimes H$ is connected if and only if either $G$ or $H$ contains an odd cycle.

Proof. Let the vertex sets of $G$ and $H$ be $\left\{p_{i}\right\}$ and $\left\{q_{j}\right\}$ respectively. Let $q_{1}$ and $q_{r}$ be adjacent vertices in $H$ and assume that $G \otimes H$ is connected. Hence there exists $\left(p_{1} q_{1} \rightarrow p_{1} q_{r}\right)=\left(p_{1} q_{1}, p_{2} q_{2}, \cdots, p_{u} q_{v}, p_{1} q_{r}\right)$ in $G \otimes H$. Associate with each vertex in this chain a pair of non- 
negative integers, $p_{i} q_{j} \sim\left(\alpha_{i}, \beta_{j}\right)$ with $\alpha_{i}$ equal to the length of a shortest chain from $p_{i}$ to $p_{1}$ in $G$ and $\beta_{j}$ the length of a shortest path from $q_{j}$ to $q_{r}$ in $H$. Clearly $p_{1} q_{1} \sim(0,1)$ and $p_{u} q_{v} \sim(1,1)$. If $p_{w} q_{x}$ and $p_{y} q_{z}$ are adjacent vertices in the chain above then $\left|\alpha_{y}-\alpha_{w}\right| \leqq 1$ and $\left|\beta_{z}-\beta_{x}\right| \leqq 1$. For otherwise the minimality of the chains would be violated. If at each step in the path both components experience a nonzero change then the sums $\alpha_{i}+\beta_{j}$ would have the same parity. But since $p_{1} q_{1} \sim(0,1)$ and $p_{u} q_{v} \sim(1,1)$ it follows that at one step in the chain either $\alpha_{y}-\alpha_{w}=0$ or $\beta_{z}-\beta_{x}=0$. Let $\beta_{z}-\beta_{x}=0$. Then there are two minimal chains $q_{z} \rightarrow q_{r}$ and $q_{x} \rightarrow q_{r}$. Let $q_{m}$ be the first vertex common to both of these chains. Now $n\left(q_{x} \rightarrow q_{m}\right)=n\left(q_{z} \rightarrow q_{m}\right)$. For otherwise the original chain would not be minimal. Hence the cycle $\left(q_{z} \rightarrow q_{m}, q_{m} \rightarrow q_{x}, q_{z}\right)$ is odd. The same argument applies if $\alpha_{y}=\alpha_{w}$. Therefore either $G$ or $H$ contains an odd cycle.

Conversely, let $G$ contain an odd cycle. Let $p_{1} q_{1}$ and $p_{2} q_{2}$ be arbitrary vertices of $G \otimes H$. Since $G$ and $H$ are connected, there are chains $p_{1} \rightarrow p_{2}$ and $q_{1} \rightarrow q_{2}$ in $G$ and $H$ respectively. If $n\left(p_{1} \rightarrow p_{2}\right)$ $+n\left(q_{1} \rightarrow q_{2}\right)$ is even then from Lemma 1 it follows that $p_{1} q_{1}$ is connected to $p_{2} q_{2}$ in $G \otimes H$. Suppose $n\left(p_{1} \rightarrow p_{2}\right)+n\left(q_{1} \rightarrow q_{2}\right)$ is odd. Let $p_{1} \rightarrow p_{1}$ be a chain which includes exactly one odd cycle of $G$. Then $n\left(p_{1} \rightarrow p_{1}\right)$ is odd and since $n\left(p_{1} \rightarrow p_{1}, p_{1} \rightarrow p_{2}\right)=n\left(p_{1} \rightarrow p_{1}\right)+n\left(p_{1} \rightarrow p_{2}\right)$, it follows that $n\left(p_{1} \rightarrow p_{1}, p_{1} \rightarrow p_{2}\right)+n\left(q_{1} \rightarrow q_{2}\right)$ is even. Hence $p_{1} q_{1}$ is connected to $p_{2} q_{2}$ in $G \otimes H$. A similar argument holds if we assume that $H$ contains an odd cycle.

COROLLARY. If $G$ and $H$ are conrected graphs with no odd cycles then $G \otimes H$ has exactly two connected components.

Proof. If $q_{1}$ is adjacent to $q_{2}$ in $H$ then $p_{1} q_{1}$ and $p_{1} q_{2}$ are not connected. For if they were it would follow that either $G$ or $H$ had an odd cycle, contrary to hypothesis. Let $p_{k} q_{k}$ be an arbitrary vertex of $G \otimes H$. It will suffice to show that $p_{k} q_{k}$ is connected either to $p_{1} q_{1}$ or $p_{1} q_{2}$. Consider chains $p_{k} \rightarrow p_{1}$ and $q_{k} \rightarrow q_{1}$. If $n\left(p_{k} \rightarrow p_{1}\right)+n\left(q_{k} \rightarrow q_{1}\right)$ is even then by Lemma $1 p_{k} q_{k}$ is connected to $p_{1} q_{1}$. Otherwise consider the chain $\left(q_{k} \rightarrow q_{1}, q_{2}\right) . n\left(q_{k} \rightarrow q_{1}, q_{2}\right)=n\left(q_{k} \rightarrow q_{1}\right)+1$ and hence $n\left(p_{k} \rightarrow p_{1}\right)$ $+n\left(q_{k} \rightarrow q_{1}, q_{2}\right)$ is even. It therefore follows from Lemma 1 that $p_{k} q_{k}$ is connected to $p_{1} q_{2}$. This completes the proof of the Corollary.

II. If $G$ is a disconnected graph then $G$ may be represented by a tecomposed matrix

$$
A=\left(\begin{array}{ll}
C & 0 \\
0 & D
\end{array}\right)
$$


with $C$ and $D$ square matrices. We will say that an adjacency matrix $M$ is decomposable if there exists a permutation matrix $P$ such that $P M P^{-1}$ is decomposed. Hence Theorem 1 gives a necessary and sufficient condition that the Kronecker product of two adjacency matrices be indecomposable. If this condition, the existence of an odd cycle, can be characterized matrix-wise then Theorem 1 can be restated purely in matrix terms. We now proceed to do this.

Lemma 2. A graph $G$ contains an odd cycle if and only if it possesses an adjacency matrix

$$
A=\left(\begin{array}{ll}
C & * \\
* & *
\end{array}\right)
$$

with $C$ an odd order matrix each row of which contains exactly two nonzero entries.

Proof. Let $G$ be a graph with vertex set $\left\{p_{i}\right\}$ which contains an odd cycle. Let $p_{i} \rightarrow p_{i}$ be an odd cycle of minimal length in $G$. Renumber the vertices of $G$ choosing the vertices of $p_{i} \rightarrow p_{i}$ as the first $k$ and the rest arbitrarily. Let $A$ be the associated adjacency matrix. Then

$$
A=\left(\begin{array}{ll}
C & * \\
* & *
\end{array}\right)
$$

with $C$ of order $k$, and every row of $C$ contains at least two nonzero entries. If any row were to contain more than two nonzero entries then there would exist an odd cycle composed of the vertices represented by $C$ which contained less than $k$ vertices, contradicting the minimality of $k$. Hence every row contains exactly two nonzero entries.

Conversely, if $A$ is an adjacency matrix of the form

$$
\left(\begin{array}{ll}
C & * \\
* & *
\end{array}\right)
$$

with $C$ an odd order matrix containing exactly two nonzero entries in every row then $C$ is an adjacency matrix of a graph containing an odd cycle. For if every vertex of a graph is adjacent to exactly two others then, neglecting multiple connections, the graph is composed of a set of disconnected cycles. But since $C$ has odd order one of these cycles must be odd.

Hence we may restate Theorem 1. 
Theorem 1'. Let $A$ and $B$ be two indecomposable adjacency matrices. Then the Kronecker product $A \otimes B$ is indecomposable if and only if there exists either $P_{1}$ or $P_{2}$, permutation matrices, such that

$$
P_{1} A P_{1}^{-1}=\left(\begin{array}{ll}
C & * \\
* & *
\end{array}\right)
$$

with $C$ an odd order matrix containing exactly two nonzero entries in every row or

$$
P_{2} B P_{2}^{-1}=\left(\begin{array}{ll}
D & * \\
* & *
\end{array}\right)
$$

with $D$ similarly defined.

\section{REFERENCES}

1. D. König, Theorie der endlichen und unendlichen graphen, Akademie Verlag M.B.H., Leipzig, 1936.

UNIVERSITY OF ILLINOIS 